\title{
Synthèse de monoglycérides par transestérification catalysée par des oxydes basiques
}

The synthesis of monoglycerides through the catalysed transesterification of basic oxides

Oléagineux, Corps Gras, Lipides. Volume 8, Numéro 3, 253-7, Mai - Juin 2001, Fondamental

Auteur(s) : Sébastien BANCQUART, Céline VANHOVE, Yannick POUILLOUX, Joël BARRAULT, Laboratoire de catalyse en chimie organique, UMR CNRS 6503, ESIP, 40, avenue de Recteur-Pineau, 86022 Poitiers Cedex, France.

Résumé : La préparation de monoglycérides à partir d'acides ou d'esters méthyliques gras et de glycérol peut être effectuée en présence de catalyseurs acides ou basiques. L'emploi de catalyseurs basiques solides pourrait limiter les réactions secondaires menant à la dégradation des réactifs, notamment du glycérol. Une comparaison de plusieurs solides basiques ( $\mathrm{MgO}, \mathrm{CeO} 2$, La2O3 et $\mathrm{ZnO}$ ) a montré que plus la basicité intrinsèque du catalyseur est importante, plus il est actif. Afin d'augmenter les performances de ces solides, plusieurs méthodes ont été utilisées pour la préparation de $\mathrm{MgO}$. Un dopage alcalin a ensuite été tenté afin d'augmenter la basicité et l'activité de MgO. L'oxyde de magnésium préparé par hydratation d'un oxyde commercial et dopé au lithium, suivi de sa déshydratation, est le catalyseur le plus actif.

Mots-clés : glycérol, transestérification, oxyde de magnésium, ester gras.

Summary : The preparation of monoglycerides from fatty acids or fatty methyl esters and glycerol can be carried out in the presence of acid or basic catalysts. The use of solid basic catalysts could limit secondary reactions leading to product degradation. A comparison of several basic solids ( $\mathrm{MgO}$, $\mathrm{CeO} 2, \mathrm{La} 2 \mathrm{O} 3$ and $\mathrm{ZnO}$ ) has shown, that the more significant the intrinsic basicity is, the more active the catalyst is. In order to increase the performance of these solids, several methods have been used for the preparation of $\mathrm{MgO}$. Alkaline doping enhanced both the basicity and the activity of $\mathrm{MgO}$. Finally, MgO prepared by hydration followed by calcination of a commercial raw material and doped by $L i$ is the most active catalyst for the preparation of glycerol mono and distearate.

Keywords : glycerol, transesterification, magnesium oxide, fatty ester.

\section{ARTICLE}

Les principaux composants des huiles végétales sont les triglycérides, ou triesters de glycérol. Leur valorisation industrielle consiste généralement en une hydrolyse ou une méthanolyse afin d'obtenir les acides ou esters méthyliques gras. Or, cette opération génère une forte production de glycérol, qui doit être rentabilisée. L'une des utilisations possibles du glycérol est la synthèse de monoglycérides (ou monoesters de glycérol), tensioactifs (ou précurseurs de) dont l'intérêt industriel 
se justifie par la large gamme de HLB accessible ainsi que par leur biocompatibilité. Les monoglycérides ont ainsi un intérêt particulier dans la synthèse de médicaments, de produits cosmétiques, de détergents, etc. [1].

Les monoglycérides peuvent être obtenus par :

- glycérolyse des triglycérides ;

- hydrolyse des triglycérides ;

- estérification du glycérol par des acides gras ;

- transestérification du glycérol par des esters méthyliques gras.

Bien que cette méthode implique deux étapes de synthèse (méthanolyse des huiles, puis transestérification du glycérol), c'est cette dernière voie que nous avons décidé d'étudier. En effet, les esters méthyliques sont facilement disponibles à un prix raisonnable.

Industriellement, ce sont des acides ou des bases utilisés en milieu homogène qui catalysent cette réaction (soude, acide paratoluène sulfonique), donnant un mélange de mono, di et triglycérides (respectivement 40, 50 et $10 \%$ ). II est donc nécessaire de mettre en œuvre une coûteuse distillation moléculaire afin d'obtenir un monoglycéride pur. En outre, il y a également nécessité de traitement des sels résultant de la transformation des catalyseurs homogènes.

Notre objectif est d'effectuer la transestérification du glycérol grâce à des catalyseurs solides basiques en absence de solvant, afin de réduire la formation de déchets et d'éliminer une étape d'extraction. Nous présentons ici l'activité catalytique de plusieurs catalyseurs solides présentant $a$ priori un caractère basique $\left(\mathrm{MgO}, \mathrm{ZnO}, \mathrm{La}_{2} \mathrm{O}_{3}\right.$ et $\left.\mathrm{CeO}_{2}\right)$.

\section{Partie expérimentale}

\section{Mesure de la basicité}

Le catalyseur (environ $65 \mathrm{mg}$ ) est préalablement dégazé sous hélium $\left(30 \mathrm{ml}^{\mathrm{min}}{ }^{-1}\right)$ pendant $8 \mathrm{~h}$ à 450 ${ }^{\circ} \mathrm{C}\left(10^{\circ} \mathrm{C} \cdot \mathrm{min}^{-1}\right)$. Puis, on injecte sur celui-ci, à intervalles de temps réguliers et à température ambiante, des pulses de dioxyde de carbone jusqu'à saturation. L'adsorption de ce gaz est suivie par un détecteur à conductivité thermique couplé à un enregistreur. Après $20 \mathrm{~min}$ sans pulse, on injecte de nouveau des pulses de $\mathrm{CO}_{2}$ jusqu'à saturation afin de déterminer la quantité de $\mathrm{CO}_{2}$ physisorbée (de manière réversible). La quantité de dioxyde de carbone réellement adsorbée par le catalyseur est la différence entre la quantité consommée et la quantité physisorbée.

Enfin, la force des sites est évaluée par désorption en augmentant la température ( $5^{\circ} \mathrm{C}$ par minute) jusqu'à $600^{\circ} \mathrm{C}$. 


\section{Mesure de l'acidité}

Le catalyseur (environ $100 \mathrm{mg}$ ) est, dans un premier temps, prétraité à $500{ }^{\circ} \mathrm{C}\left(10^{\circ} \mathrm{C} \cdot \mathrm{min}^{-1}\right)$ sous hélium $\left(40 \mathrm{ml} \cdot \mathrm{min}^{-1}\right)$. Après refroidissement à $50^{\circ} \mathrm{C}$, l'installation est purgée au moyen d'une pompe à vide primaire. Le catalyseur est ensuite saturé par de l'ammoniac, l'excès d'ammoniac étant éliminé par dégazage sous vide.

La désorption peut alors être réalisée, sous hélium $\left(40 \mathrm{ml} \cdot \mathrm{min}^{-1}\right)$, en élevant la température par paliers de $50{ }^{\circ} \mathrm{C}\left(4{ }^{\circ} \mathrm{C} \cdot \mathrm{min}^{-1}\right)$ et celle-ci est suivie par détection catharométrique. La quantité totale d'ammoniac désorbé est déterminée en faisant passer le courant gazeux $\left(\mathrm{He}-\mathrm{NH}_{3}\right)$ dans une solution d'acide chlorhydrique $10^{-2} \mathrm{~N}$. L'enregistrement des pics de désorption permet de déterminer les quantités d'ammoniac désorbé à chaque température et ainsi d'établir une distribution des sites acides selon l'énergie d'adsorption de l'ammoniac.

\section{Préparation de $\mathrm{MgO}$ (II) par précipitation}

On dissout $100 \mathrm{~g}$ de nitrate de magnésium $\mathrm{Mg}\left(\mathrm{NO}_{3}\right)_{2}, 6 \mathrm{H}_{2} \mathrm{O}$ dans $250 \mathrm{ml}$ d'eau distillée. Sous agitation, $100 \mathrm{ml}$ d'une solution d'ammoniaque (environ $30 \%$ ) sont ajoutés. Le précipité, ainsi obtenu, est lavé plusieurs fois à l'eau distillée afin d'éliminer les ions nitrates $\mathrm{NO}_{3}{ }^{-}$puis séché à l'étuve $\left(100{ }^{\circ} \mathrm{C}\right)$ pendant $16 \mathrm{~h}$. L'oxyde de magnésium est ensuite obtenu par calcination sous air $\left(40 \mathrm{ml}^{-\mathrm{h}^{-1}}\right)$ à $450{ }^{\circ} \mathrm{C}$ $\left(5^{\circ} \mathrm{C} \cdot \mathrm{min}^{-1}\right)$ pendant $12 \mathrm{~h}$.

\section{Préparation de MgO (III) par hydratation/déshydratation}

À $25 \mathrm{~g}$ d'oxyde de magnésium commercial (Magchem 25), on ajoute goutte à goutte $250 \mathrm{ml}$ d'eau distillée sous agitation, puis on chauffe à $80^{\circ} \mathrm{C}$ toujours sous agitation pour éliminer l'excès d'eau. On obtient ainsi une pâte épaisse qui est chauffée à $85^{\circ} \mathrm{C}$ sur bain de sable jusqu'à obtention d'un solide sec. Enfin, ce dernier est calciné sous air à $350{ }^{\circ} \mathrm{C}$ (montée en température : $3^{\circ} \mathrm{C} / \mathrm{min}$ ) pendant $2 \mathrm{~h}$ puis à $500^{\circ} \mathrm{C}$ (montée en température : $5^{\circ} \mathrm{C} / \mathrm{min}$ ) pendant $8 \mathrm{~h}$.

\section{Test catalytique}

La réaction est effectuée dans un tricol en pyrex de $125 \mathrm{ml}$ équipé :

- d'un agitateur mécanique (hélice et axe en acier inox) ;

- d'un Dean-Stark (extracteur de méthanol) associé à un réfrigérant ;

- d'une arrivée d'azote couplée à un puits thermométrique, cette arrivée servant aussi d'ouverture pour le prélèvement des échantillons.

Le ballon, calorifugé par de la laine de verre, est porté à la température désirée.

L'ester méthylique et le glycérol sont portés à $220^{\circ} \mathrm{C}$ sous agitation d'environ 500 tours. $\min ^{-1}$, puis le catalyseur est ajouté, donnant le temps zéro de la réaction.

La réaction est réalisée sous atmosphère d'azote afin d'éviter l'oxydation de l'ester méthylique et du glycérol. 


\section{Résultats}

\section{Comparaison des oxydes}

Les oxydes métalliques utilisés ont été caractérisés afin de quantifier leur acidité et leur basicité ainsi que leur surface spécifique (tableau 1). II apparaît alors que $\mathrm{ZnO}$ et $\mathrm{CeO}_{2}$ sont dominés par leurs propriétés acides, tandis que $\mathrm{MgO}$ est dominé par ses propriétés basiques. Le cas de l'oxyde de lanthane est plus complexe : la présence de carbonates et d'oxycarbonates de lanthane, qui se décomposent à haute température, fausse la mesure en surestimant les propriétés basiques [2-4].

Ces oxydes ont été testés dans la réaction de transestérification du glycérol par le stéarate de méthyle, en rapport équimolaire, sans solvant. Les résultats sont présentés dans le tableau 2.

Les résultats montrent des comportements très disparates, mais il semble que l'activité catalytique est liée à la basicité intrinsèque du solide (figure 1).

Deux solides ( $\mathrm{MgO}$ et $\mathrm{La}_{2} \mathrm{O}_{3}$ ) semblent donc être de bons concurrents pour se substituer aux catalyseurs homogènes. Cependant, en présence de $\mathrm{La}_{2} \mathrm{O}_{3}$, on observe, vraisemblablement à cause de ses propriétés acides, la formation d'acroléine, fortement toxique et devant donc être évitée. L'étude a donc été poursuivie avec MgO.

\section{Influence de la méthode de préparation de l'oxyde de magnésium}

Afin d'augmenter l'activité de l'oxyde de magnésium, plusieurs méthodes de synthèse ont été envisagées. Les caractéristiques physicochimiques de trois oxydes de magnésium se trouvent dans le tableau 3 : MgO (I) est un oxyde commercial de Prolabo, $\mathrm{MgO}$ (II) est préparé par une précipitation à partir de nitrate de magnésium et d'ammoniaque, selon la méthode décrite par Berkani et al. [5], tandis que $\mathrm{MgO}$ (III) est préparé par hydratation-déshydratation de MgO (I).

Il apparaît que la méthode de préparation de l'oxyde de magnésium a une forte influence sur les caractéristiques physicochimiques du solide obtenu, notamment sur sa surface spécifique. Néanmoins, dans tous les cas, l'oxyde de magnésium présente majoritairement des propriétés basiques.

Ces trois oxydes ont été testés pour la réaction de transestérification du glycérol par le stéarate de méthyle. Les activités initiales sont données dans le tableau 4.

Ainsi, l'activité de l'oxyde de magnésium est liée à la surface spécifique du solide utilisé, même s'il n'y a pas proportionnalité directe entre ces variables. L'activité intrinsèque augmente également avec la basicité intrinsèque, comme indiqué précédemment. La méthode (III), hydratation-déshydratation de MgO (I), donne ainsi les meilleurs résultats. Cependant, il faut remarquer que l'estimation de I'activité moyenne d'un site basique est plus élevée avec le solide MgO (II). II sera donc intéressant d'examiner plus particulièrement la nature des sites basiques résultant du changement de la méthode de préparation. On constate que l'utilisation d'un précurseur identique conduit à la même activité moyenne du catalyseur, $\mathrm{MgO}$ (I) et $\mathrm{MgO}$ (III). 


\section{Dopage par des éléments alcalins}

La surface de l'oxyde de magnésium est normalement constituée d'un réseau d'ions $\mathrm{Mg}^{2+}$ et $\mathrm{O}_{2}$. Lorsqu'on dope un oxyde de magnésium par un élément alcalin, on échange une partie des ions $\mathrm{Mg}^{2+}$ par des ions $\mathrm{M}^{+}$, où $\mathrm{M}$ représente un alcalin ( $\mathrm{Li}, \mathrm{K}, \mathrm{Na}, \mathrm{Rb}, \mathrm{Cs}$ ). Ceci nécessite la formation de sites « $\mathrm{O}^{\prime}$ » afin de préserver l'électroneutralité du réseau. Les ions $\mathrm{M}^{+}$stabilisent ainsi les $\mathrm{O}^{-}$par la création d'espèces [ $\left.\mathrm{M}^{+} \mathrm{O}^{-}\right][6,7]$. Celles-ci sont responsables de l'augmentation de la basicité du matériau. La littérature donne quelques exemples d'utilisation d'oxyde de magnésium dopé comme catalyseur, notamment l'utilisation par Lunsford et son équipe [8] dans la réaction de couplage oxydant du méthane. Choudhary et al. [9] ont montré grâce à cette réaction l'influence de l'élément alcalin introduit. Ainsi, $\mathrm{Na} / \mathrm{MgO}$ est plus basique que $\mathrm{Li} / \mathrm{MgO}$, lui-même plus basique que MgO (à concentration molaire et non massique en alcalin identique).

Des essais ont donc été réalisés avec des oxydes de magnésium contenant environ $1 \%$ (en poids) d'alcalin. La diffraction des rayons $X$ a confirmé dans tous les cas la conservation de la structure cristallographique « MgO ». Les caractéristiques physico-chimiques de ces solides sont données dans le tableau 5.

L'addition d'un alcalin provoque donc la diminution de la surface spécifique. Celle-ci serait dépendante du pourcentage atomique d'alcalin ajouté. En outre, l'addition d'un alcalin augmente à la fois la basicité intrinsèque et l'acidité intrinsèque (par unité de surface) de l'oxyde. Il est à noter que le caractère basique est effectivement le plus marqué pour l'oxyde de magnésium dopé au lithium. En effet, pour les autres oxydes dopés, on observe des rapports A/B supérieurs à 1 .

Parmi les éléments étudiés, le lithium est celui qui modifie le plus la structure du MgO, vraisemblablement en raison de la taille voisine des ions $\mathrm{Li}^{+}$et $\mathrm{Mg}^{2+}$ (tableau 6).

Les oxydes dopés ont été utilisés dans la réaction de transestérification du glycérol par le stéarate de méthyle. Les résultats sont donnés dans le tableau 7.

Il apparaît ainsi que tous les oxydes de magnésium dopés sauf l'oxyde de magnésium dopé au lithium sont moins actifs que l'oxyde de magnésium seul, ce qui confirme le fait que l'activité du catalyseur augmente avec sa basicité intrinsèque (figure 2).

\section{Sélectivité}

Les répartitions en mono, di et triglycérides obtenues en présence d'oxyde de lanthane et d'oxyde de magnésium ont été comparées (figure 3).

Nous n'observons pas de différence significative de sélectivité en fonction du catalyseur étudié. De plus, nous retrouvons des sélectivités (à $80 \%$ de conversion) comparables aux sélectivités obtenues en milieu homogène ( $40 \%$ de monoglycérides et $50 \%$ de diglycérides). Ceci est tout à fait normal du fait que les catalyseurs utilisés ne sont pas capables d'induire ce que nous appelons en catalyse hétérogène une "sélectivité de forme ". Des études sont en cours actuellement pour modifier ce catalyseur afin de lui permettre d'induire cette sélectivité. 


\section{CONCLUSION}

Ce travail montre que des oxydes métalliques solides peuvent substituer des catalyseurs homogènes classiques dans les réactions de trans-estérification du glycérol. Leur activité est directement liée à leur basicité. Cependant, des matériaux comportant également des propriétés acides $\left(\mathrm{La}_{2} \mathrm{O}_{3}\right.$ par exemple) favorisent également la déshydratation du glycérol en acroléine, ce qui n'est pas recommandé.

Des solides étudiés, MgO apparaît comme le meilleur candidat : fortement basique et peu acide, il ne favorise pas la formation d'acroléine tout en gardant une activité convenable. De plus, il a été montré que la méthode de préparation de l'oxyde de magnésium avait une grande influence sur ses propriétés physico-chimiques, et donc sur son activité, le plus actif étant l'oxyde de magnésium préparé par hydratation-déshydratation de l'oxyde de magnésium commercial. On a pu constater également que la nature des sites basiques était très certainement fonction de la méthode de préparation. Enfin, des essais réalisés avec des oxydes de magnésium dopés ont montré que le solide le plus efficace était l'oxyde de magnésium dopé au lithium.

Cette étude prouve donc que l'activité du solide utilisé comme catalyseur est bien fonction de sa basicité intrinsèque. Cependant, tous les centres identifiés comme basiques ne sont certainement pas actifs dans notre réaction et il nous faut maintenant mieux caractériser cette basicité.

Les solides étudiés n'ayant pas la possibilité d'induire une sélectivité de forme, nous n'observons pas de différence notable de sélectivité en monoglycérides par rapport à la réaction en milieu homogène. Des études sont en cours pour obtenir des catalyseurs à base d'oxyde de magnésium plus sélectifs.

\section{Remerciements}

Ce travail a été réalisé dans le cadre du programme européen AIR n 3CT 942218 " Réactivité des esters gras et du glycérol : nouvelles méthodes " et les auteurs remercient la Communauté européenne. Ils remercient également la stéarinerie Dubois et l'Ademe pour leur soutien à la fois scientifique et financier.

\section{REFERENCES}

1. RIEGER MM (1990). Glyceryl stearate, chemistry and use. Cosmetics and Toiletries, 105 : 51-7.

2. PAK S, SMITH E, ROSYNEK MP, LUNSFORD JH (1997). Conversion of methyl radicals to methanol and formaldehyde over vanadium oxide catalysts. J Catal, $165: 73-9$.

3. TAYLOR RP, SCHRADER GL (1991). Lanthanum catalysts for CH oxidative coupling : a comparison of the reactivity of phases. Ind Eng Chem Res, $30: 1016-23$.

4. LE VAN T, CHE M, TATIBOUET JM, KERMAREK M (1993). Infrared study of the formation and stability of $\mathrm{La}_{2} \mathrm{O}_{2} \mathrm{CO}_{3}$ during the oxidative coupling of methane on $\mathrm{La}_{2} \mathrm{O}_{3}$. J Catal, $142: 18-26$.

5. BERKANI M (1995). Réduction de composés carbonylés par transfert d'hydrogène sur catalyseurs solides. Thèse de l'université de Poitiers. 
6. JOHNSON MA, STEFANOVICH EV, TRUONG T (1997). An ab initio study on the oxidative coupling of methane over a lithium-doped MgO catalyst : surface defects and mechanism. J Phys Chem B 101 : 3196-201.

7. DI COSIMO JI, DIEZ VK, APESTEGUIA CR (1996). Base catalysis for the synthesis of alpha, betaunsaturated ketones from the vapor-phase aldol condensation of acetone. Appl Catal A, 137 : 14966.

8. ITO T, LUNSFORD JH (1985). Synthesis of ethylene and ethane. Oxidation of methane over lithium doped magnesium oxide. Nature, $314:$ 721-2.

9. CHOUDHARY VR, RANE VH (1990). Influence of various promoters on the basicity and catalytic activity of MgO catalysts in oxidative coupling of methane. Catal Lett, 6 : 95-8.

Illustrations

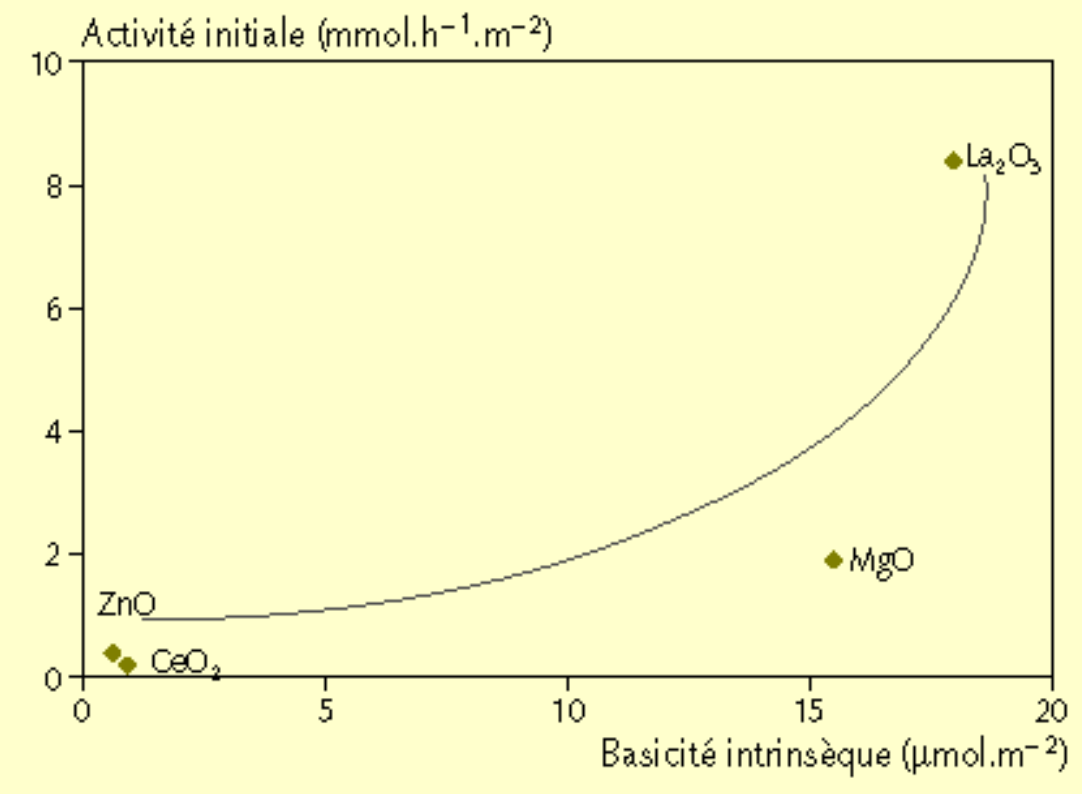

Figure 1. Activité initiale des oxydes métalliques dans la réaction de transestérification du glycérol par le stéarate de méthyle en fonction de la basicité intrinsèque. 


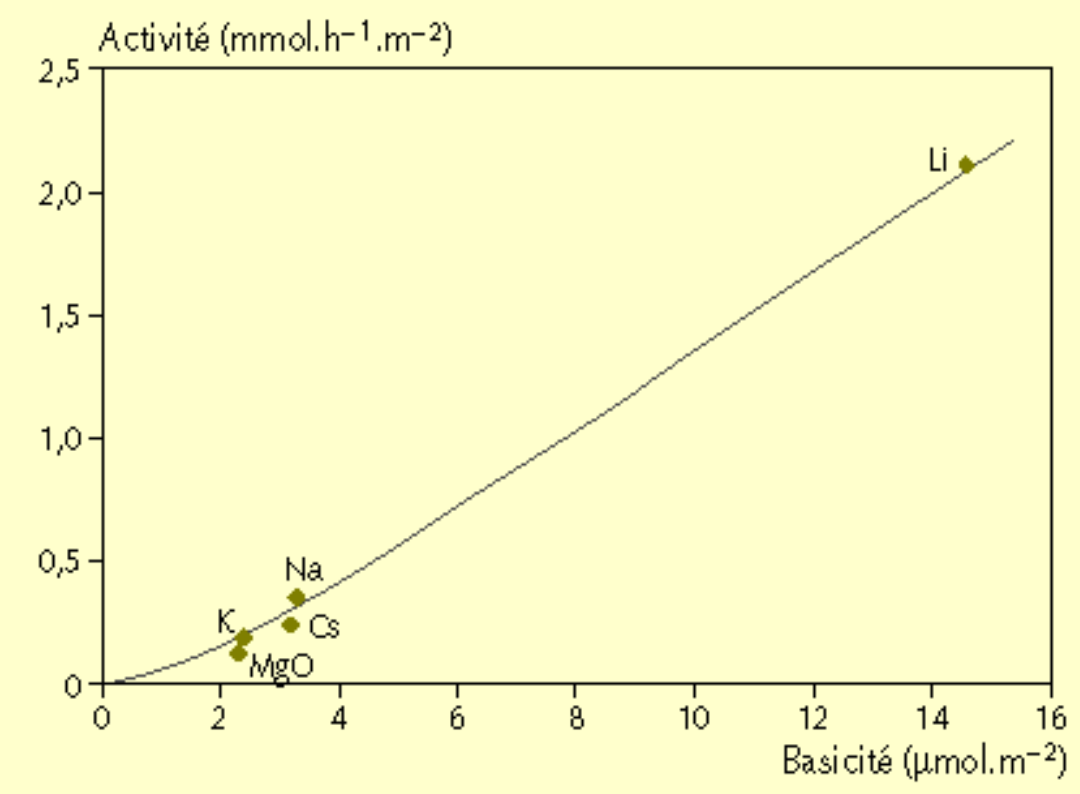

Figure 2. Activité initiale des oxydes métalliques dopés dans la réaction de transestérification du glycérol par le stéarate de méthyle en fonction de la basicité intrinsèque. 


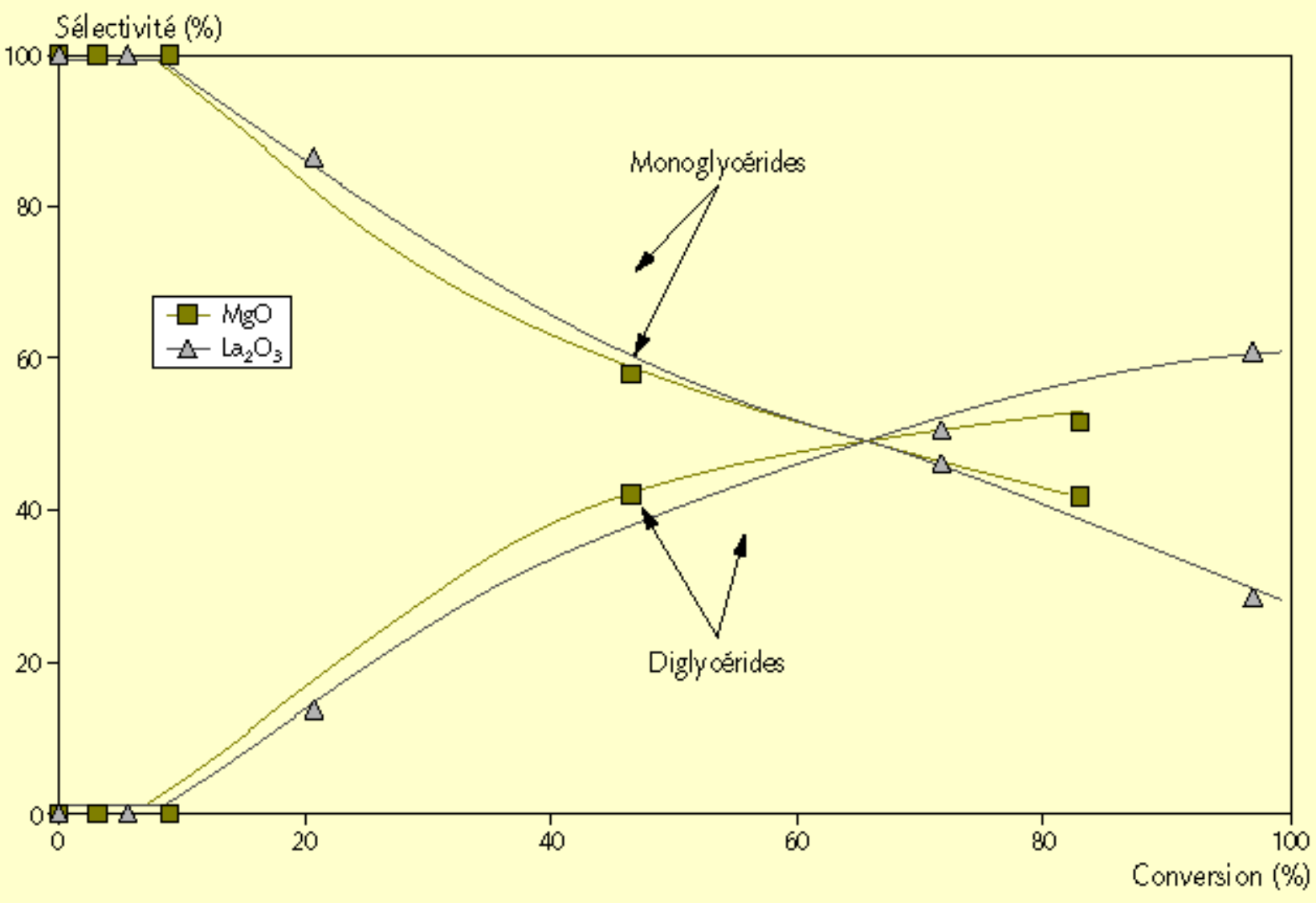

Figure 3. Transestérification du glycérol par le stéarate de méthyle. Répartition des produits formés.

Tableau 1. Caracterisation des oxydes utilises par desorption en temperature programmee de $\mathrm{CO}_{2}$ et de $\mathrm{NH}_{\mathrm{J}^{\prime}}$

\begin{tabular}{|c|c|c|c|c|c|c|}
\hline \multirow[t]{2}{*}{ Catalyseur } & \multirow{2}{*}{$\begin{array}{c}S_{1 \mathrm{II}} \\
\left(m^{2} \cdot g^{-1}\right)\end{array}$} & \multicolumn{2}{|c|}{ Acidit6 } & \multicolumn{2}{|c|}{ Basicite } & \multirow[t]{2}{*}{$A / B^{d}$} \\
\hline & & $\mu \mathrm{mol} . \mathrm{g}^{-1}$ & $\mu \mathrm{mol} . \mathrm{m}^{-2}$ & $\mu \mathrm{mol} \cdot \mathrm{g}^{-1}$ & $\mu \mathrm{mol} . \mathrm{m}^{-2}$ & \\
\hline $\mathrm{ZnO}^{\mathrm{a}}$ & 33,0 & 455 & 13,9 & 21,0 & 0,6 & 23,2 \\
\hline$M g O^{\infty}$ & 13,0 & 110 & 8.5 & 202,0 & 15,5 & 0,5 \\
\hline $\mathrm{La}_{2} \mathrm{O}_{3}{ }^{\mathrm{D}}$ & 4,6 & 565 & 120,0 & 80,6 & 18,0 & 6,7 \\
\hline $\mathrm{CeO}_{2}^{\mathrm{c}}$ & 19,0 & 546 & 29,0 & 16,3 & 0,9 & 32,2 \\
\hline
\end{tabular}

a : obtenu par calcination de carbonate de zinc sous air pendant $4 \mathrm{~h}$ a $350^{\circ} \mathrm{C} .{ }^{\circ}$ : Prolabo. ${ }^{\mathrm{c}}:$ : Rhone-Poulenc. a : rapport acidite/basicite. 
Tableau 2. Conversion et selectivite en mono, di and triglycerides pour la reaction de transesterification du glycerol par k stearate de methyle en presence d'oxydes metalliques a $220^{\circ} \mathrm{C}$ apres $6 \mathrm{~h}$.

\begin{tabular}{lrrrr}
\hline Catalyseur & Conversion & \multicolumn{3}{c}{ Sélectivite (\%) } \\
\cline { 3 - 5 } & (\%) & Mono & Di & Tri \\
Sans & 2,5 & 100 & 0 & 0 \\
$\mathrm{ZnO}$ & 18,0 & 80 & 20 & 0 \\
$\mathrm{MgO}$ & 83,0 & 42 & 52 & 6 \\
$\mathrm{La}_{2} \mathrm{O}_{3}$ & 97,0 & 28 & 61 & 11 \\
$\mathrm{CeO}_{2}$ & 4,0 & 100 & 0 & 0 \\
Catalyseur & & 40 & 50 & 10 \\
homogene & 90,0 & & & \\
\hline
\end{tabular}

Atmosphere azote ; $\mathrm{T}=6 \mathrm{~h}, 220^{\circ} \mathrm{C}$, rapport stearate/glyœrol $=1$.

Tableau 3. Proprietes physicochimiques des oxydes de magnesium etudies.

\begin{tabular}{|c|c|c|c|c|c|c|c|}
\hline \multirow{2}{*}{ Catalyseur } & \multirow{2}{*}{$\begin{array}{c}S_{\text {ве }} \\
\left(m^{2} \cdot g^{-1}\right)\end{array}$} & \multirow{2}{*}{$\begin{array}{c}\text { Structure } \\
\text { DRX }\end{array}$} & \multicolumn{2}{|c|}{ Acidite } & \multicolumn{2}{|c|}{ Basicite } & \multirow{2}{*}{ A/B } \\
\hline & & & $\mu \mathrm{mol} . g^{-1}$ & $\mu \mathrm{mol} . \mathrm{m}^{-2}$ & emol.g & emol. $\mathrm{m}^{-2}$ & \\
\hline MgO (I) & 13 & $\mathrm{MgO}$ & 110 & 8,5 & 202 & 15,5 & 0,5 \\
\hline $\mathrm{MgO}(\mathrm{II})$ & 102 & $\mathrm{MgO}$ & - & - & 138 & 1,3 & - \\
\hline MgO (III) & 151 & $\mathrm{MgO}$ & 290 & 1,9 & 345 & 2,3 & 0,8 \\
\hline
\end{tabular}

Tableau 4. Activite d'oxydes de magnesium (a $20 \%$ de conversion) dans la reaction de transesterification du glycerol par le stearate de methyle. Influence de la methode de preparation.

\begin{tabular}{|c|c|c|c|c|c|}
\hline Catalyseur & $\begin{array}{c}\text { Activite } \\
\text { spécifique } \\
\left(\mathrm{mmol} \cdot \mathrm{h}^{-1} \cdot \mathrm{g}^{-1}\right)\end{array}$ & $s_{\text {IE }}\left(m^{2} \cdot g^{-1}\right)$ & $\begin{array}{c}\text { Activite } \\
\text { intrins eque } \\
\left(\mathrm{mmol}^{-1} \mathrm{~h}^{-1} \cdot \mathrm{m}^{-2}\right)\end{array}$ & $\begin{array}{c}\text { Basicite } \\
\text { intrinsèque } \\
\left(\mu \mathrm{mol} . \mathrm{m}^{-2}\right)\end{array}$ & $\begin{array}{c}\text { Activité } \\
\text { par site basique } \\
\left(h^{-1}\right)\end{array}$ \\
\hline $\mathrm{MgO}(\mathrm{I})$ & 10,7 & 13 & 0,88 & 15,5 & 57 \\
\hline $\mathrm{MgO}(\mathrm{II})$ & 14,9 & 102 & 0,15 & 1,3 & 115 \\
\hline MgO (III) & 20,2 & 151 & 0,13 & 2,3 & 57 \\
\hline
\end{tabular}

Tableau 5. Caracteristiques physico-chimiques des oxydes utilises.

\begin{tabular}{|c|c|c|c|c|c|c|c|c|}
\hline \multirow{2}{*}{ Catalyseur } & \multicolumn{2}{|c|}{ Teneur en a lcalin } & \multirow{2}{*}{$\begin{array}{c}S_{\text {เе }} \\
\left(m^{2} \cdot g^{-1}\right)\end{array}$} & \multicolumn{2}{|c|}{ Acidite } & \multicolumn{2}{|c|}{ Basicité } & \multirow{2}{*}{ A/B } \\
\hline & $\%$ massique & $\%$ atomique & & $\mu \mathrm{mol} . \mathrm{g}^{-1}$ & $\mu \mathrm{mol} . \mathrm{m}^{-2}$ & $\mu \mathrm{mol} . \mathrm{g}^{-1}$ & $\mu \mathrm{mol} . \mathrm{m}^{-2}$ & \\
\hline MgO (III) & - & - & 151,0 & 290 & 1,9 & 345 & 2,3 & 0,8 \\
\hline $\mathrm{Li} / \mathrm{MgO}$ & 0,90 & 0,130 & 20,8 & 240 & 11,5 & 303 & 14,6 & 0,8 \\
\hline $\mathrm{Na} / \mathrm{MgO}$ & 0,56 & 0,020 & 50,6 & 457 & 10,8 & 168 & 3,3 & 3,3 \\
\hline $\mathrm{K} / \mathrm{MgO}$ & 0,73 & 0,020 & 92,9 & 500 & 5,4 & 228 & 2,4 & 2,2 \\
\hline $\mathrm{Cs} / \mathrm{MgO}$ & 0,60 & 0,004 & 112,0 & 441 & 3,9 & 363 & 3,2 & 1,2 \\
\hline
\end{tabular}


Tableau 6. Rayons ioniques de $\mathrm{Mg}^{2+}$ et des elements alcalins.

\begin{tabular}{|cc}
\hline Ions & Rayon ionique (nm) \\
\hline $\mathrm{Mg}^{2+}$ & 0,066 \\
$\mathrm{Li}^{+}$ & 0,068 \\
$\mathrm{Na}^{+}$ & 0,097 \\
$\mathrm{~K}^{+}$ & 0,133 \\
$\mathrm{CS}^{+}$ & 0,167 \\
\end{tabular}

Tableau 7. Transesterification du glycerol par le stearate de methyle en presence d'oxyces de magnesium dopes. Influence de l'akalin.

\begin{tabular}{|lcccc|}
\hline Catalyseur & $\begin{array}{c}\text { Alcalin } \\
\text { (\% poids) }\end{array}$ & $\begin{array}{c}\text { Activite } \\
\text { specifique } \\
\left(\mathrm{mmol}^{-1} \cdot \mathrm{g}^{-1}\right)\end{array}$ & $\begin{array}{c}\text { Activite } \\
\text { intrinseque } \\
\left(\mathrm{mmol}^{-1} \cdot \mathrm{m}^{2}\right)\end{array}$ & $\begin{array}{c}\text { Rendement } \\
\text { en monoglycerides } \\
(\%)\end{array}$ \\
\hline $\mathrm{MgO}$ (III) & & 20,2 & 0,13 & 32,6 \\
Li/MgO & 0,90 & 44,2 & 2,10 & 33,0 \\
$\mathrm{Na} / \mathrm{MgO}$ & 0,56 & 17,9 & 0,35 & 30,6 \\
K/MgO & 0,73 & 17,9 & 0,19 & 30,6 \\
CS/MgO & 0,60 & 26,8 & 0,24 & 21,1
\end{tabular}

Conditions experimentales : rapport molaire gly/sm $=1, \mathrm{~T}=220^{\circ} \mathrm{C}$, m catalyseur $=0,5 \mathrm{~g}, \mathrm{t}=3 \mathrm{~h}$, azote, pression atmospherique. 